\title{
COMBINATIONS OF ISOTHIOCYANATES WITH DRUGS - A CHANCE OR THREAT TO CHEMOPREVENTION AND CANCER TREATMENT?
}

\author{
LIDIA MIELCZAREK ${ }^{1,2 *}$, ZDZISŁAW CHILMONCZYK ${ }^{1}$, PIOTR SUCHOCKI², \\ PIOTR WROCZYŃSKI ${ }^{2}$ and KATARZYNA WIKTORSKA ${ }^{1 *}$
}

'Department of Drug Biotechnology and Bioinformatics, National Medicines Institute, Warsaw,
32/34 Chełmska Street, 00-725 Warsaw, Poland
${ }^{2}$ Chair of Physical Pharmacy and Bioanalysis Faculty of Pharmacy, Medical University of Warsaw,

1 Banacha Street, 02-097 Warsaw, Poland

\begin{abstract}
Isothiocyanates (ITCs) are a group of compounds of natural origin which exhibit anticancer properties. In addition to the cytotoxic impact on cancer cells, confirmed in the multiple cell lines and the in vivo models, ITCs exhibit the cytoprotective effect in normal cells by regulating the activity of enzymes involved in xenobiotic metabolism. These properties of ITCs have led to a continuing increase in the number of studies which have shown that ITCs can sensitize cancer cells to cytostatic drugs used as standard in cancer therapies. On the other hand, these compounds may decrease the effectiveness of drugs by deregulating the metabolizing system of the cell. This paper discusses the results of a preclinical study on ITCs applications in combination therapy as well as their role in drug metabolism.
\end{abstract}

Keywords: isothiocyanates; combination therapy; drugs interaction; drugs combination; sulforaphane; chemoprevention; chemotherapy, drug metabolism

In recent decades, the development of pharmacotherapy, the spread of haemodynamic laboratories, and the improvement of the cardiac surgery safety profile have made the cardiovascular mortality significantly reduced. At the same time, the problem of the increasing number of cancer-related deaths has emerged. According to the World Health Organization (WHO) data in 2012, about 14 million new cases were reported and 8 million people died of proliferative diseases. Estimates for 2030 assume that these figures will be 26 million and 17 million, respectively (1).

The best therapeutic effects are still being achieved through radical, often mutilating surgeries or aggressive chemotherapy, which itself can induce another cancer. Despite these actions, the percentage of people who are fully cured is still unsatisfactory. For this reason, in recent years, researchers are more and more interested in combination therapy to maximize efficacy and simultaneously reduce adverse effects of the treatment.

In 2005 a new research trend has emerged in the scientific literature, according to which the activity of oncological drugs can be enhanced by natural compounds with the confirmed antitumor activity, such as genistein, resveratrol, turmeric and isothiocyanates (ITCs).

ITCs are compounds naturally occurring in the vegetables of the Brassicaceae family, such as Brussels sprouts, cabbage, cauliflower and broccoli. Numerous studies dating back to the 1990's indicate that ITCs affect various signalling pathways important in the process of inhibiting the tumor growth; they modify carcinogen metabolism, stop tumor cell proliferation, angiogenesis and metastasis and induce apoptosis (2-9) (Fig. 1). In vitro and in vivo studies indicate that ITCs protect against the development of many malignancies, including colorectal, prostate, breast, lung cancer, and leukemia. The most widely studied compound of this group is sulforaphane (1-isothiocyanato-4-methylsulfinyl-butane, SFN). The anticancer activity has also been demonstrated by SFN derivatives, such as benzyl isothiocyanate (BITC), 2-phenylethyl isothiocyanate (PEITC), allyl isothiocyanate (AITC) and erysolin (Fig. 2). The multi-directional activity of

\footnotetext{
* Corresponding author: e-mail: 1.mielczarek@nil.gov.pl, e-mail: k.wiktorska@nil.gov.pl
} 
ITCs and the awareness of ineffectiveness and toxicity of conventional therapies have been the basis for attempts to use ITCs as adjuvants for standard anticancer therapy. However, due to an ability to modulate the activity of I, II and III phase xenobiotic metabolism enzymes, ITCs can interact with many drugs, including anticancer drugs.

The aim of the article is to summarize the studies on ITCs applications in combination therapy as well as their role in drug metabolism conducted for over 25 years and to draw conclusions on the effects of these compounds on the activity of anticancer drugs and drug metabolizing enzymes.

\section{Negative influence of ITCs on drug effectiveness}

The studies showed that ITCs may attenuate the effectiveness of many drugs. It is mainly associated with induction of phase II and phase III enzymes of xenobiotic metabolism in normal and transformed cells. The research conducted by Leslie et al. demonstrated that ITCs induced the expression of MRP1 and MRP2 (multidrug resistance-associated protein 1 and 2) transporting proteins simultaneously contributing to the excessive excretion of drugs from the cell, such as vincristine or doxorubicin (DOX), resulting in the reduced therapeutic response to the treatment (10). De Larco et al. have also shown that ITCs reduced paclitaxel toxicity towards MCF-7 breast cancer cells in which the expression of MRP and NQO1(NAD(P)H quinone dehydrogenase 1) proteins was observed (11). The recent research conducted by Zeekpudsa et al. indicates that the excessive expression of NQO1 in cholangiocarcinoma cells (CCA) may cause resistance to treatment with compounds, such as 5-fluorouracil, DOX, or gemcitabine. The studies have also shown that NQO1 expression-deficient cells are susceptible to therapy (12).

The discussed data strongly indicate that the ITCs impact on drug metabolizing enzymes in particular NQO1 and MRP may lead to attenuate the cytostatic drug effectiveness. The induction of these enzymes was also observed in other cell lines. For example, the studies performed by Maher et al.

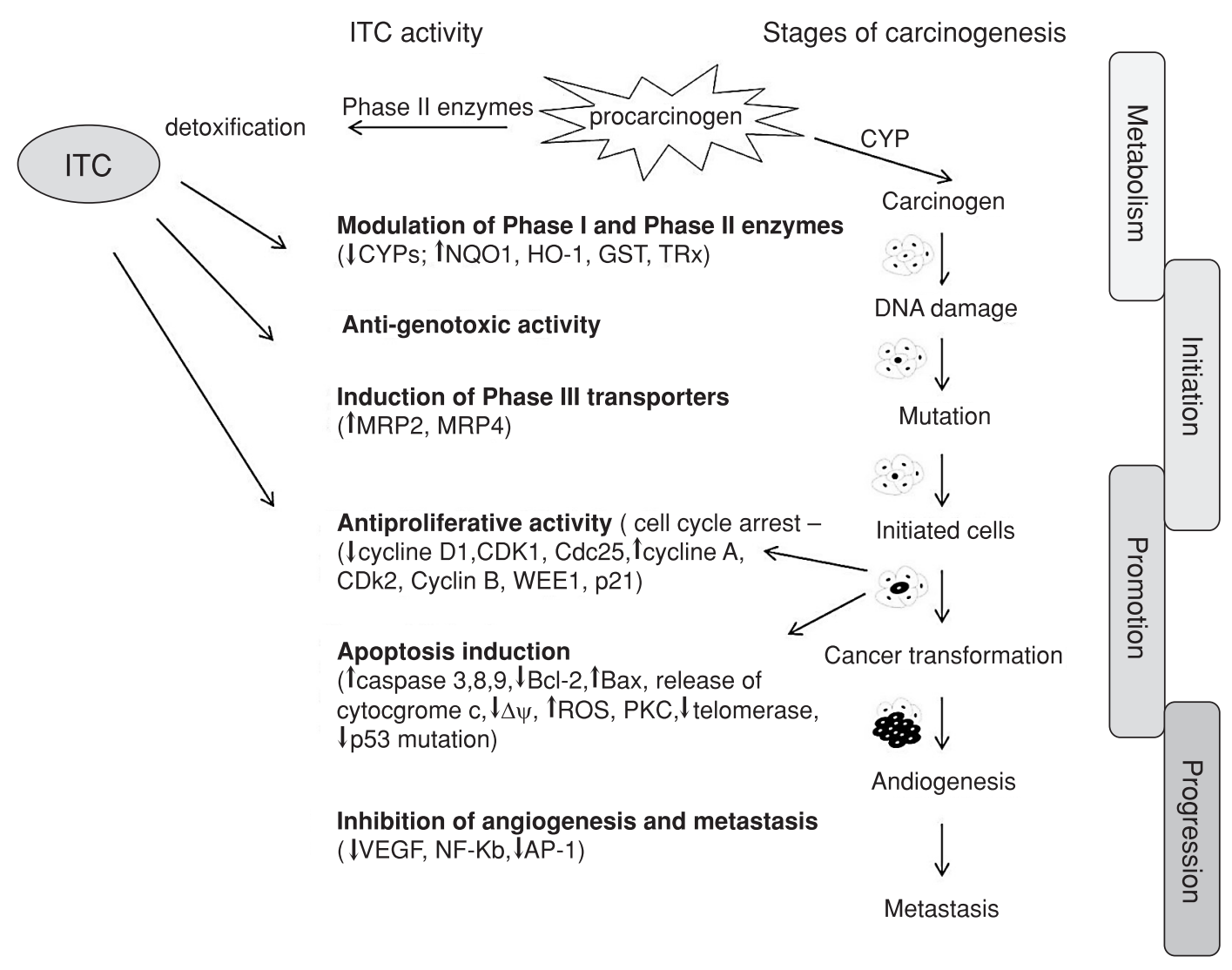

Figure 1. The anticancer activity of ITCs molecular mechanism of action 
<smiles>CS(=O)CCCCN=C=S</smiles>

Sulforaphane<smiles>C=CCN=C=S</smiles>

Allyl Isothiocyanate<smiles>CS(=O)(=O)CCCCN=C=S</smiles>

Erysolin<smiles>S=C=NCCc1ccccc1</smiles>

2-Phenylethyl Isothiocyanate<smiles>S=C=NCc1ccccc1</smiles>

Benzyl Isothiocyanate<smiles>S=C=Nc1cccc2ccccc12</smiles>

$\alpha$-Naphtyl Isothiocyanate

Figure 2. Chemical structures of some ITCs used in drug interaction studies

showed that SFN induced NQO1 and MRPs expression in normal and transformed hepatic cell lines (13). Moreover, Harris et al. proved that SFN increased MRP levels in Caco-2 and A549 cell lines (14).

Interesting results were also presented by Lubelska et al. This research showed that SFN and alyssin could act as antagonists of frequently used medications (including OTC drugs) like ketoprofen, verapamil and furosemide with respect to their impact on the metabolizing enzymes in Caco-2 colon cancer cells. Performed studies indicated, that ITC (administered together with furosemide, verapamil or ketoprofen) may alter drug metabolism by enhancing the gene expression and activity of the following enzymes involved in drug metabolism and transport: belonging to the II phase of xenobiotic metabolism - NQO1, GSTA1, GSTA3 (glutathione S-transferases 1 and 3 ) and to the III phase - a transport protein MRP1. Described phenomenon may lead to changes in drug efficacy and the development of multidrug resistance (MDR) $(15,16)$.

\section{Augmentation of drug effectiveness by ITC - the influence of ITCs on the activity of phase I, II, III xenobiotic metabolism enzymes}

The literature data indicate also that ITC may increase therapeutic activity of drugs by affecting the cellular enzymatic system involved in drug metabolism. The research conducted by Zhou et al. has shown that by inhibiting CYP3A4 - key drug activating enzyme belonging to I phase of xenobiotic metabolism, SFN may lead to a reduction in adverse effect of drugs, such as rifampicin or midazolam. The induction of CYP3A4, among others, by drugs like rifampicin or midazolam can cause adverse therapeutic response (e.g., the lack of efficacy), which is a serious therapeutic problem. Hence, these studies are an important prerequisite for the development of new therapeutic and dietary therapies in order to reduce the incidence of adverse drug reactions (17).

Reinicke et al. emphasized important fact, that the activity of II phase enzymes is often required to convert pro-drugs into their active forms. For instance, the NQO1 activity is required to convert beta-lapachone derivatives into their therapeutically active form. Low NQO1 expression may cause failure of antineoplastic treatment with the compound mentioned above, as was shown in breast, pancreatic, and non-small cell lung cancer (18).

The newest publication of Qazi et al. have demonstrated that SFN inhibited the activity of MRP transport protein and thus increased the suppression of esophageal adenocarcinoma cell growth (BEAC) by paclitaxel (19). In studies conducted by Tseng et al., SFN analogs (BITC, PEITC, phenyl-, 1naphthyl- (NITC), phenyl-hexyl-, phenylpropyl-, and phenylbutyl-ITC, erucin, and erysolin) (Fig. 2) inhibited PGP (P-glycoprotein) or MRP1-dependent excretion of daunorubicin and vinblastine from MCF-7/ADR breast cancer cells and PANC-1 pancreatic cancer cells. It has been shown that this led to the greater accumulation of these compounds in cells, including those resistant to daunorubicin (MCF-7/ADR), and thereby the increased effectiveness of therapy (20).

\section{ITCs in combination - chemoprevention}

Apart of the known data on ITC activity as a chemopreventive agent, the recent literature deliver 
examples of the promising effect of combinations of ITCs in the field of chemoprevention. The significant effect of ITCs in combination with selenium compounds have been observed in normal cells. Davis et al. have assessed the impact of the supplementation with selenium-enriched broccoli at the dose over $800 \mu \mathrm{g} / \mathrm{g}$, while the average selenium content in broccoli was less than $1 \mu \mathrm{g} / \mathrm{g}$. Studies have shown that the consumption of selenium-enriched broccoli lyophilisate reduced the incidence of both small and large intestine cancer in APC gene-loaded mice, whose presence predisposes to these tumors. In addition, a significant increase has been reported in the selenium levels in blood and liver of animals, along with the increased activity of antioxidant enzyme GPx (glutathione peroxidase) in erythrocytes compared with the administration of selenium alone (Table 1) (21).

In turn, the study conducted in rats by Blum et al. measured the level of antioxidant enzymes GPx in the small and large intestine, as well as in the liver after the combined administration of glucoraphanin (SFN precursor) and selenium. The use of the combination, compared to the separate administration, resulted in a significant increase in the level of antioxidant enzymes, including GPx1 and GPx2 in each of the examined organs. The increase of the expression of enzymes was associated with the activation of the NFE2L2/KEAP1 (Nuclear factor (erythroid-derived 2)-like 2/kelch like ECH associated protein 1) pathway. The studies indicate an increased anticancer potential of the combination compared to the single use of the compounds (Table 1) (22).

The anticancer action of SFN-selenium combination has also been demonstrated in EAhy926 vascular epithelial cells. The incubation of cells with selenium alone (IV) resulted in an increase in the activity of antioxidant enzymes, such as TRx (Thioredoxin), GPx1 and GPx4. In contrast, SFN increased the activity of cytoplasmic Thioredoxin (TRx1). The combination of SFN and selenium (IV) resulted in the higher expression of TRx1-encoding genes as compared to the separate administration. The combination also increased endothelial cell protection against oxidative stress caused by tert-butyl hydroperoxide (t-BuOOH) (23).

Other studies have also confirmed the synergistic impact of the combination of selenium compounds and SFN derivatives on increasing the GPx and TRx1 activity, and thus protecting normal cells against carcinogens and oxidative stress-induced death. The elevation of the TRx level has been demonstrated in studies on immortalized human
HHL-2 hepatocytes as well as in CCD841 stem cells line after the administration of sodium selenite and SFN. The mechanism of SFN action was based on the induction of the synthesis of TRx mRNA. Simultaneously, selenium played a structural role as a component of the enzyme and protected from TRx mRNA degradation (Table 1) $(4,24,25)$.

At the same time, recent research on the combined use of SFN and its analogs (alyssin, 2-oxohexyl ITC and 2-oxoheptyl ITC) with the organic selenium compound -Selol has shown the enhanced anticancer effect of the combination in HT-29 colorectal cancer cells. Studies have shown that ITCs can to varying degrees potentiate the cytotoxic effect of Selol. The effect of Selol was most significantly strengthened by SFN, resulting in the synergism- mutual enhancement of the properties of the tested compounds. The other results indicate that the combination of Selol and SFN analogs have an additive effect (according to the Chou-Talalay method) (Table 1) (7). The research provides the basis for further studies on the combination of selenium and ITCs compounds in relation to another cell lines.

\section{ITCs in anticancer combined therapy}

Drug resistance to anticancer compounds has long been a major clinical problem. The properties of SFN and ITCs explain the attempts to use these compounds in the combined therapy. Up to date, in vitro studies have shown that ITCs can sensitize tumor cells to cytostatic drugs used as a standard in cancer therapies. Synergism has been demonstrated for ITCs and, among others, cisplatin (3, 9, 26), gemcitabine, doxorubicin (DOX), 5-fluorouracil (5FU) $(5,8)$, etoposide (6), paclitaxel (27) and oxaliplatin (28). The most important results of studies on the use of ITCs with anticancer compounds are summarized in Table 1.

\section{ITCs and arsenic}

Arsenic trioxide (Arsenic, ATO) is a compound used in the treatment of acute promyelocytic leukemia. In vitro studies have also demonstrated its cytotoxic and proapoptotic activity against myeloid leukemia, lymphoma, multiple myeloma and lymphoma (29).

Studies conducted in acute myeloid leukemia (HL-60) and chronic myelogenous leukemia (K562) cell lines have demonstrated that ITCs: SFN and erysolin increase the cytotoxic ATO activity. The use of these combinations resulted in the rapid growth of reactive oxygen species (ROS) in cancer cells, with the consequent increase in ATO-induced 


\begin{tabular}{|c|c|c|c|c|c|c|c|c|c|c|}
\hline 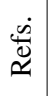 & & $\begin{array}{l}\text { oे } \\
\text { ci }\end{array}$ & & હ્రీ & $\stackrel{d}{d}$ & 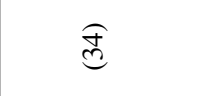 & $\widehat{\mathscr{C}}$ & & $\begin{array}{l}\hat{\sigma} \\
\hat{\sigma}\end{array}$ & \\
\hline 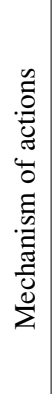 & 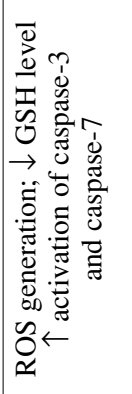 & 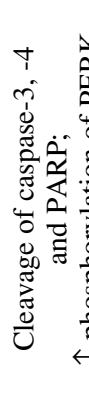 & 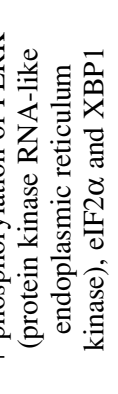 & $\overleftrightarrow{\mathbb{z}}$ & 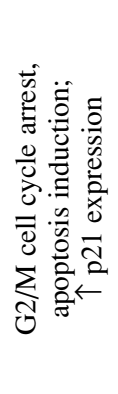 & 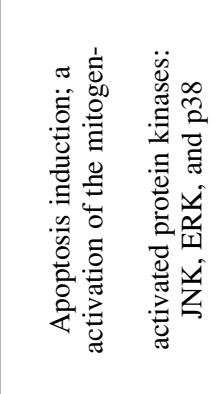 & 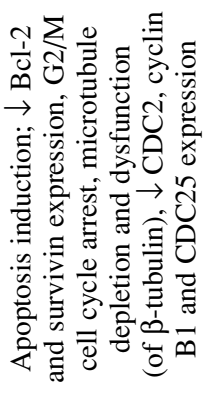 & 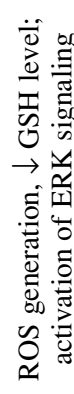 & 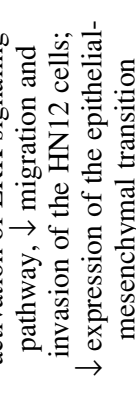 & 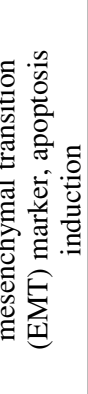 \\
\hline 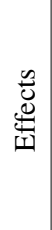 & & 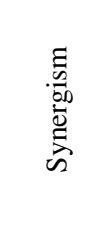 & & 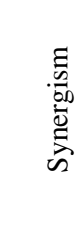 & 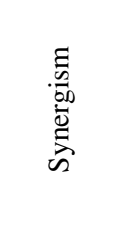 & 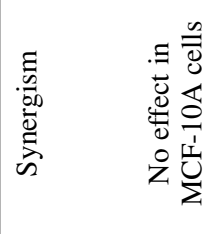 & 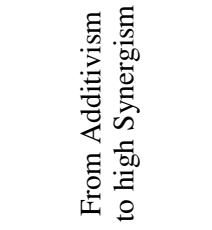 & 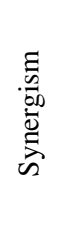 & 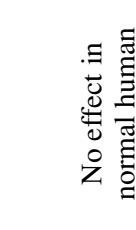 & 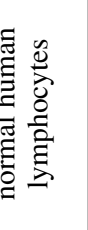 \\
\hline 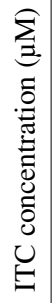 & 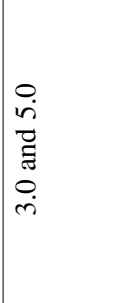 & & & 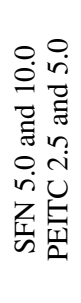 & $\begin{array}{l}0 \\
\text { ì } \\
i \\
n \\
\text { in }\end{array}$ & $\stackrel{\circ}{\dot{r}}$ & $\begin{array}{l}8 \\
8 \\
\dot{+} \\
1 \\
1 \\
0 \\
0 \\
0 \\
0\end{array}$ & $\begin{array}{l}0 \\
\text { in } \\
\text { E } \\
\text { in } \\
n \\
i\end{array}$ & $\begin{array}{l}8 \\
\stackrel{0}{0} \\
1 \\
\stackrel{1}{1}\end{array}$ & \\
\hline $\begin{array}{l}\stackrel{0}{.} \\
\stackrel{\Xi}{\overline{0}}\end{array}$ & 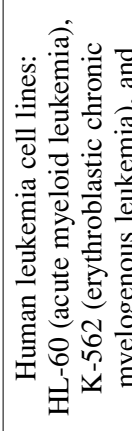 & 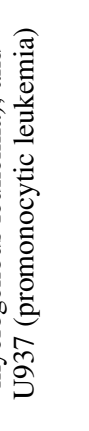 & 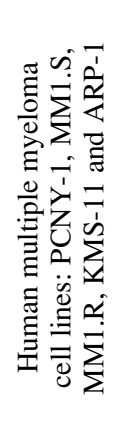 & 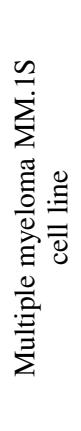 & 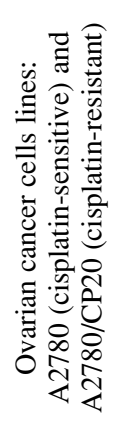 & 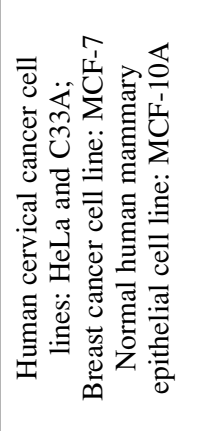 & 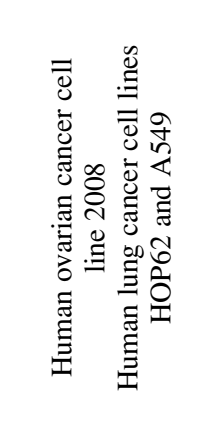 & 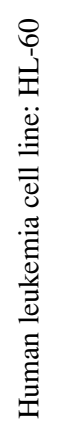 & 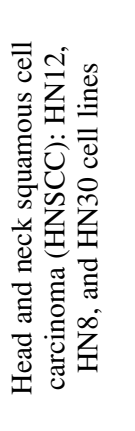 & 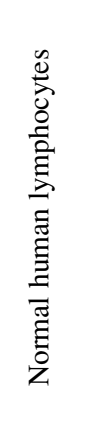 \\
\hline 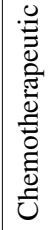 & & 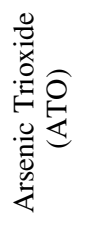 & & 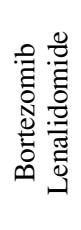 & 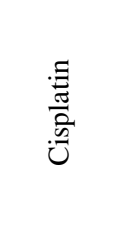 & $\begin{array}{l}\frac{7}{5} \\
\frac{7}{2} \\
\frac{2}{3} \\
0\end{array}$ & 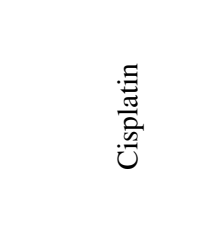 & & $\begin{array}{l}\frac{\Xi}{5} \\
\frac{5}{2} \\
\frac{2}{3} \\
0\end{array}$ & \\
\hline 氖 & 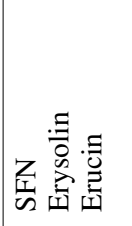 & & & 至基 & 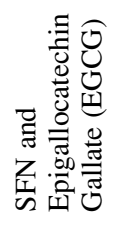 & 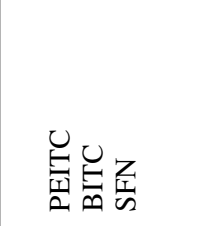 & 岁 & & $\stackrel{U}{:}$ & \\
\hline
\end{tabular}




\begin{tabular}{|c|c|c|c|c|c|c|}
\hline$\frac{\dot{m}}{\mathscr{\Delta}}$ & శ్రీ & $\widehat{\mathrm{I}}$ & $\stackrel{0}{+}$ & 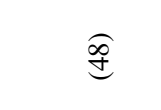 & $\begin{array}{l}\hat{n} \\
\hat{n} \\
\text { Bn }\end{array}$ & है \\
\hline 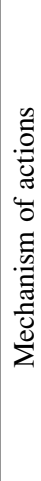 & 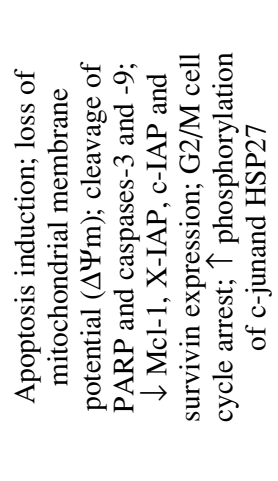 & 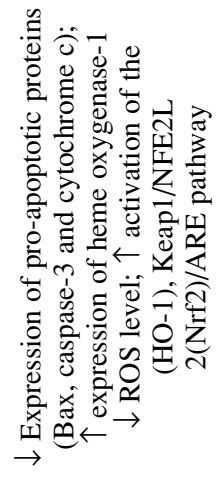 & 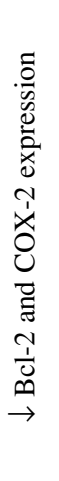 & 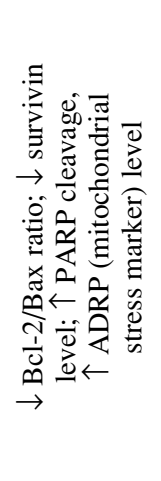 & 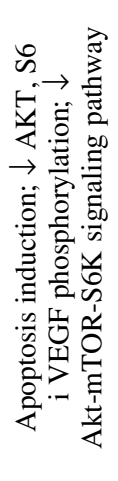 & 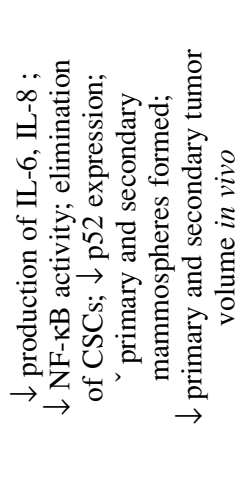 \\
\hline 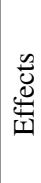 & 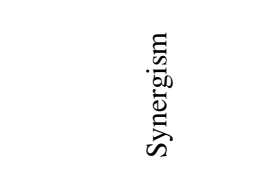 & 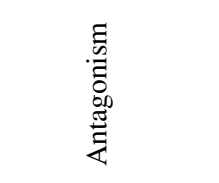 & 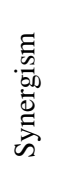 & 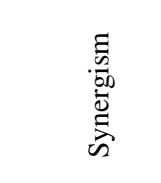 & 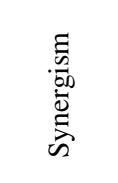 & 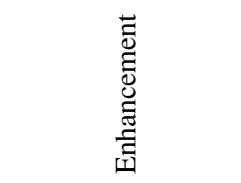 \\
\hline 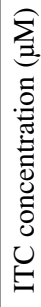 & 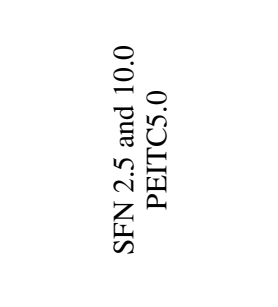 & 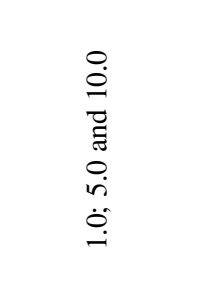 & $\begin{array}{l}8 \\
0 \\
i \\
i \\
1 \\
0 \\
0\end{array}$ & $\begin{array}{l}0 \\
\dot{n} \\
n \\
n \\
i\end{array}$ & $\begin{array}{l}\stackrel{\leftrightarrow}{\mathrm{i}} \\
\dot{n} \\
\dot{i}\end{array}$ & $\begin{array}{l}\stackrel{0}{0} \\
\dot{1} \\
\dot{i}\end{array}$ \\
\hline $\begin{array}{l}\stackrel{\mathscr{\Xi}}{\Xi} \\
\overline{\bar{\Theta}}\end{array}$ & 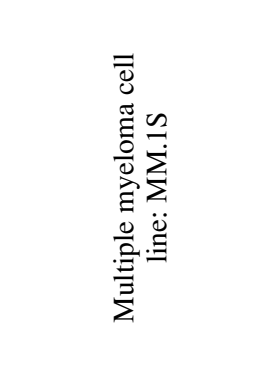 & 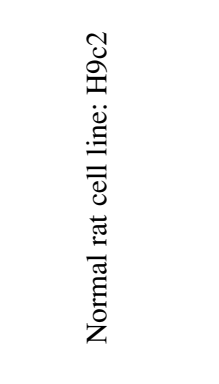 & 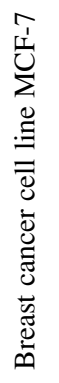 & 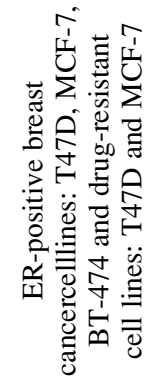 & 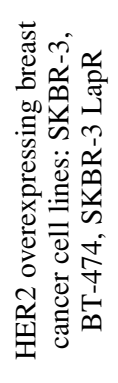 & 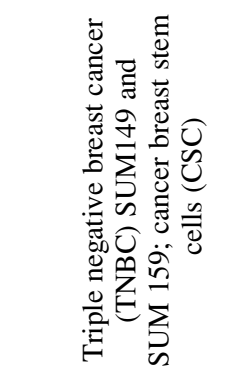 \\
\hline 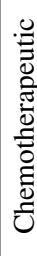 & 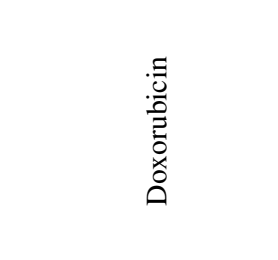 & 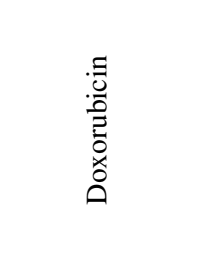 & 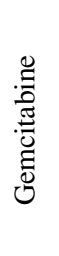 & 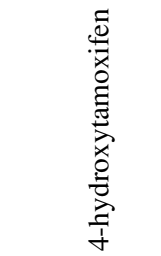 & 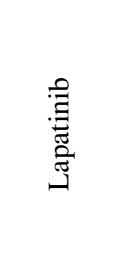 & 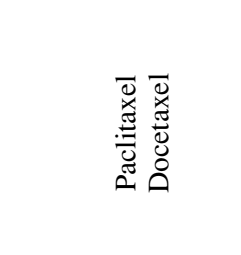 \\
\hline $\begin{array}{l}\infty \\
E\end{array}$ & 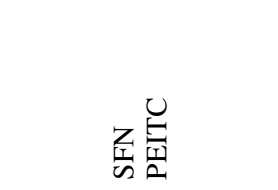 & 窝 & 至 & 尩窇 & 惡息 & 壹 \\
\hline
\end{tabular}


Combinations of isothiocyanates with drugs - a chance or...

\begin{tabular}{|c|c|c|c|c|c|c|}
\hline 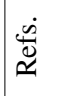 & 总 & $\hat{E}$ & $\widehat{\mathbb{d}}$ & $\begin{array}{l}\widetilde{a} \\
\text { J }\end{array}$ & \& & $\stackrel{\widehat{\infty}}{\stackrel{\infty}{n}}$ \\
\hline 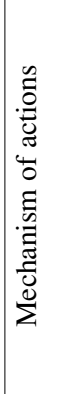 & 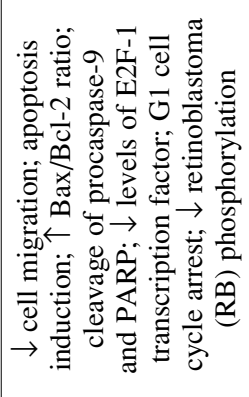 & 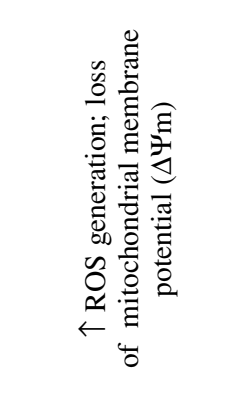 & 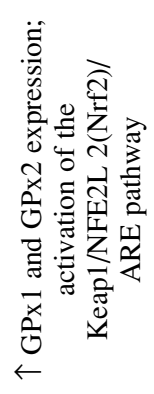 & 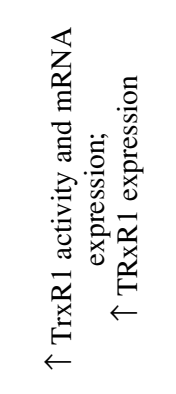 & 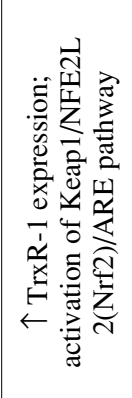 & 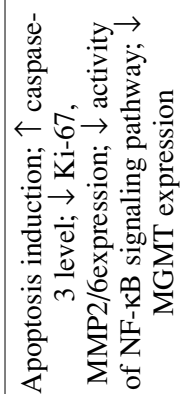 \\
\hline 竞 & 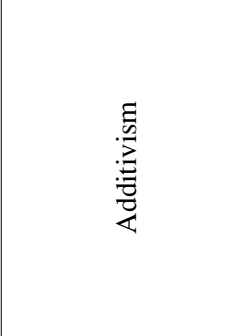 & 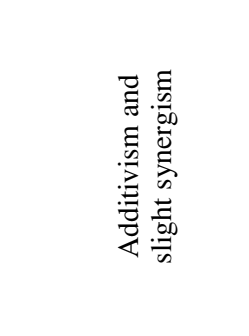 & 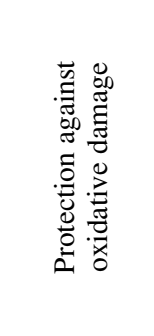 & 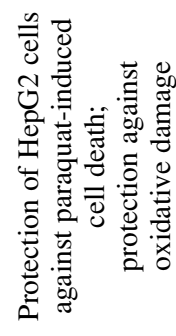 & 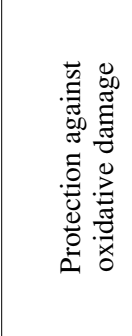 & 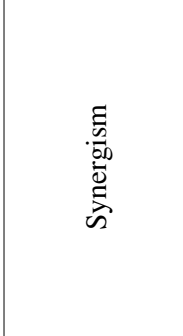 \\
\hline 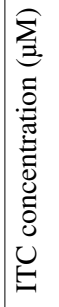 & 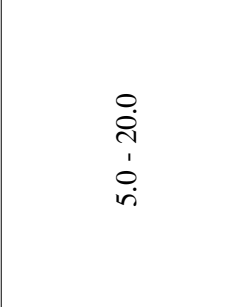 & $\begin{array}{l}\stackrel{0}{\vec{m}} \\
\dot{1} \\
\stackrel{1}{-}\end{array}$ & 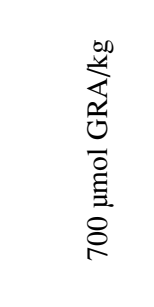 & $\simeq$ & $\frac{8}{0}$ & $\begin{array}{l}0 \\
\dot{p} \\
\dot{m} \\
\dot{0} \\
\dot{n}\end{array}$ \\
\hline$\frac{\stackrel{\mathscr{e}}{\Xi}}{\stackrel{\vec{\Xi}}{\overline{0}}}$ & 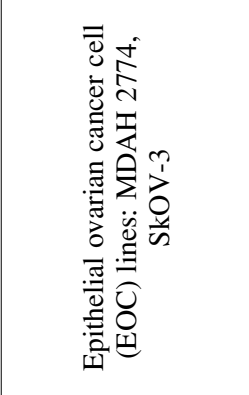 & 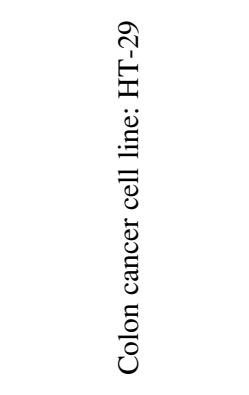 & 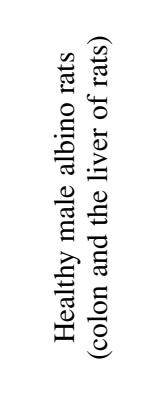 & 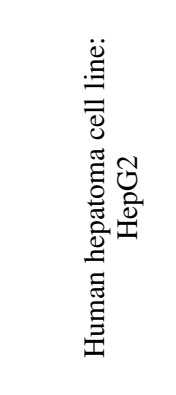 & 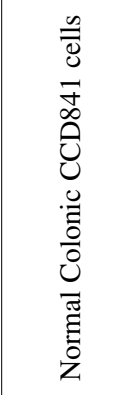 & 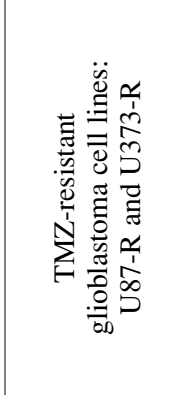 \\
\hline 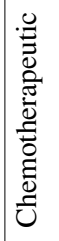 & 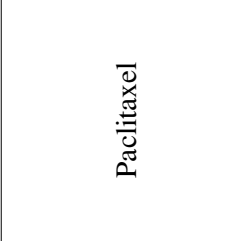 & 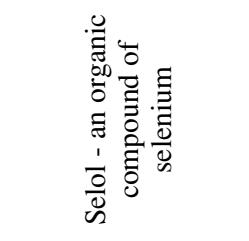 & 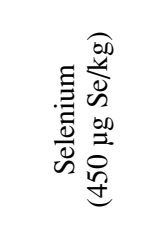 & 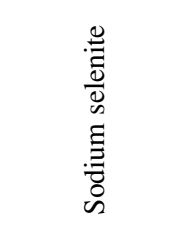 & 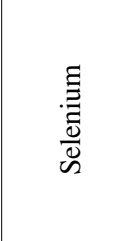 & 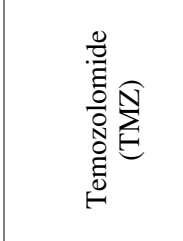 \\
\hline $\begin{array}{l}\hat{n} \\
E\end{array}$ & 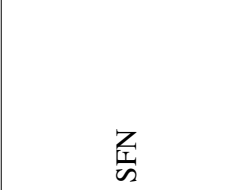 & 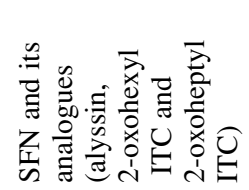 & 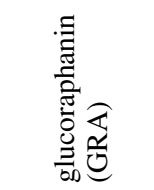 & 至 & 至 & $\underset{\text { 萦 }}{\mathrm{Z}}$ \\
\hline
\end{tabular}


apoptosis (2). Similarly, in the studies conducted by Doudican et al. SFN has been shown to sensitize multiple myeloma cells to ATO. The compounds showed the synergistic effect on apoptosis and the induction of caspase- 3 and caspase-4. The compounds used in the combination caused an increase in the cellular ROS level, resulting in the depletion of cellular glutathione levels and the consequent cell death (Table 1). The increased expression of heatshock protein HSP90 was also observed $(2,30)$.

\section{ITCs and bortezomib, lenalidomide}

Bortezomib is an anticancer agent, a selective reversible proteasome inhibitor. It inhibits the activity of $26 \mathrm{~S}$ proteasome - a large protein complex degrading proteins with the attached ubiquitin. The inhibition of $26 \mathrm{~S}$ proteasome prevents ubiquitinproteasome proteolysis which influences the signal transduction cascades in a cancer cell, ultimately leading to its apoptosis. Bortezomib is used in a variety of combinations with lenalidomide, dexamethasone, and melphalan in the treatment of multiple myeloma (31).

Pre-clinical studies conducted by Jakubikova indicate that ITCs can increase the efficacy of multiple myeloma therapy (Table 1) (32). The study aimed to investigate whether SFN (5 and $10 \mu \mathrm{M}$ ) and PEITC ( 2.5 and $5 \mu \mathrm{M})$ potentiate the effects of the aforementioned drugs in multiple myeloma (MM.1S) therapy. The study on the combination of SFN and bortezomib, SFN and dexamethasone and SFN and melphalan showed a synergistic effect of the combination across the range of concentrations used. Moderate synergism has been demonstrated for the combination of SFN and lenalidomide. In turn, for the combination of drugs with PEITC, the considerable effect was demonstrated for all tested drugs. The additive effect was shown only for the PEITC concentration $2.5 \mu \mathrm{M}$ in the combination with dexamethasone (32).

\section{ITC and cisplatin, oxaliplatin}

Cytotoxic effects of oxaliplatin or cisplatin include the formation of DNA cross-links, thus preventing replication and cell division.

The research conducted by Kaminski et al. has demonstrated the synergistic effect of SFN with oxaliplatin in the treatment of colorectal cancer. In the studies conducted in Caco-2 cells, the cytotoxic effect of oxaliplatin in combination with SFN (10 and 20 $\mu \mathrm{M}$ ) was higher compared to the alone administration of compounds. The effect was related to the lower intracellular ATP levels, caspase-3 activation, DNA fragmentation and PARP cleavage (28).
The studies conducted by Chen et al. on cisplatin sensitive (A2780) and resistant (A2780/ CP20) ovarian cancer cells have demonstrated that the use of SFN with epigallocatechin gallate (EGCG) enhanced cisplatin proapoptotic properties. In addition, the use of this combination led to a marked increase in the number of cells in G2/M cell cycle phase compared to the cells treated with cisplatin alone (Table 1) (26). Other studies showed that also AITC potentiates cisplatin effect on ovarian cancer cells (33).

The studies carried out using ITC phenylethyl and benzyl derivatives have also shown the increased cisplatin antitumor efficacy after the use of the compounds in combination. Studies on the combination of non-toxic doses of PEITC and cisplatin in HeLa cells have demonstrated that these compounds act synergistically by the activation of caspase-3 (33). This effect has also been observed in the studies on the combination of BITC and SFN with cisplatin in $\mathrm{C} 33 \mathrm{~A}$ cervical cancer cells as well as MCF-7breast cancer cells. Importantly, these combinations did not have cytotoxic effects on normal MCF-10 breast cells (Table 1) (34).

Lee et al. have shown that BEITC sensitizes head and neck squamous cell carcinoma (HNSCC) and leukemia cells (HL-60) to cisplatin cytotoxicity. It has been found that the incubation with $3 \mu \mathrm{M}$ cisplatin reduced the viability of HL-60 cells to $71 \%$, whereas the combination of $3 \mu \mathrm{M}$ cisplatin and $5 \mu \mathrm{M}$ of BITC resulted in a decrease in cell viability to $21 \%$. Importantly, this combination was not toxic to normal lymphocytes. The synergistic effect was related to a decrease in the cellular glutathione (GSH) level, ROS generation and the activation of extracellular-signal-regulated kinase (ERK) pathway $(3,9)$.

The study conducted by Di Pasqua et al. showed differences in the action of SFN, BITC and PEITC (Fig. 2) in NCI-H596 small cell lung cancer. Both BITC and PEITC have been demonstrated to sensitize lung cancer cells to cisplatin by $\beta$-tubulin degradation. Interestingly, the preincubation of cells with SFN did not give this effect (35).

Studies conducted on animals confirmed that SFN supplementation during cisplatin treatment protected normal cells from cisplatin-induced damage. It has been shown that in rats treated with cisplatin in combination with SFN, there were a smaller proportion of necrotic cells as in rats treated with the cytostatic alone (36).

\section{ITC and DOX}

DOX is an anthracycline antibiotic used alone or in combination in numerous malignancies, such 
as breast, lung, gastric, ovarian, thyroid cancer, and sarcoma. Its effect is to inhibit cancer cell proliferation through intercalation to DNA, as well as ROS generation causing lipid peroxidation, mitochondrial membrane damage and the consequent apoptosis of cancer cells (37).

Fimognari et al. have investigated the combination of SFN and DOX in murine fibroblasts with p53 protein mutation (Ser220), p53 knock-out mouse fibroblasts and p53 wild-type cells. Cells with mutations in $\mathrm{p} 53$ protein or lacking this protein are highly resistant to DOX. Studies have shown that DOX did not induce apoptosis in Ser220 cells and knock-out mouse fibroblast. Apoptosis was induced only after the administration of very high DOX concentrations $(10 \mu \mathrm{M})$. The combined administration of SFN and DOX caused cellular sensitization to DOX, the activation of caspase-3 and apoptosis. SFN also led to a sharp drop in the GSH cellular levels contributing to the increased susceptibility of cells to oxidative stress induced by DOX (38).

A similar effect was obtained in studies in which HeLa cervical cancer cells were pre-incubated with SFN or PEITC for 24 hours at concentrations of 0.1-5 $\mu \mathrm{M}$ followed by the administration of DOX and etoposide. SFN has increased the efficacy of the drug by stimulating apoptosis through the caspase- 3 and caspase- 9 activation, as well as by reducing the level of C-protein kinase (PKC) and telomerase activity in tumor cells. This enabled the reduction of the doses of the cytostatics while maintaining therapeutic efficacy, which is of great importance in the protection of normal cells against toxicity of the therapy. $\mathrm{EC}_{50} \mathrm{DOX}$ for HeLa cells was $90 \mu \mathrm{M}$. After the addition of $5 \mu \mathrm{M}$ SFN $\mathrm{EC}_{50}$ decreased to $23 \mu \mathrm{M}$; the combined administration of DOX and PEITC resulted in $\mathrm{EC}_{50}$ DOX decrease to $20 \mu \mathrm{M}(6)$.

Mukherjee et al. confirmed the mechanism of the synergistic action of DOX and PEITC in the studies conducted in PC-3 prostate cancer cells. This was due to the lowering of the antiapoptotic PKC isoforms (PKC-á and PKC-1́) and the activity of telomerase as well as inducing apoptosis by stimulating caspase- 3 and caspase- 8 . The same effect was achieved in the combination of etoposide and PEITC (39). The study conducted by Jakubikova et al. showed that $10 \mu \mathrm{M}$ SFN and 2.5 and $5 \mu \mathrm{M}$ PEITC in combination with $25 \mu \mathrm{M}$ DOX synergistically inhibited the proliferation of multiple myeloma cells (MM.1S) (32) (Table 1). The studies of the combination of SFN and doxorubicin in breast cancer cells MCF-7 and MDA-MB-231 also demon- strated synergism in inhibiting cell proliferation (40).

Importantly, the study conducted by $\mathrm{Li}$ et al. in normal H9c2 cells revealed that SFN protects normal cells against DOX cardiotoxicity. These compounds used together effectively lowered the level of free radicals by inducing HO-1 expression. The increased expression of the enzyme responsible for neutralization of ROS was achieved by the activation of the NFE2L2/KEAP1 pathway. In addition, the use of SFN-DOX significantly reduced the percentage of apoptotic cells compared to the administration of DOX alone. This has been associated with a decrease in the levels of proapoptotic Bax protein, caspase- 3 and cytochrome $\mathrm{c}$ in normal cells (Table 1) (41). These findings have been confirmed by Singh et al. who also indicated that the SFN cardioprotective activity was based on the activation of the KEAP1/NFE2L2/ARE pathway (42).

\section{ITC and 5-fluorouracil}

5-fluorouracil (5-FU) is a cytostatic antimetabolite of pyrimidine. Its action mainly involves the inhibition of thymidylate synthase. It is primarily used in the treatment of head and neck, gastrointestinal tract or breast malignancies (43).

Wang et al. have proven that SFN abolishes 5FU resistance typical for adenoid cystic carcinoma of the salivary gland. The studies were conducted using high (ACC-M) and low-invasive (ACC-2) salivary gland cancer cells. The synergistic interaction was explained by the effect of the combination on the regulation of $\mathrm{NF}-\kappa \mathrm{B}$ transcription factor (nuclear factor kappa-light-chain-enhancer of activated $\mathrm{B}$ cells). The enhanced activation of NF- $\kappa \mathrm{B}$ is responsible for the resistance to antineoplastic agents. The simultaneous use $(20 \mu \mathrm{M} \mathrm{SFN}+60 \mu \mathrm{M}$ $5-\mathrm{FU})$ decreased the viability of tumor cells and reduced the expression of $\mathrm{NF}-\mathrm{\kappa B}$ transcription factor (8).

\section{ITC and gemcitabine}

Gemcitabine is a pyrimidine cytostatic antimetabolite drug that inhibits DNA synthesis. It is mainly used to treat non-small cell lung cancer (NSCLC), pancreatic cancer, breast cancer and ovarian cancer (44).

The study conducted by Sharma C. et al. has demonstrated synergistic effects of SFN and gemcitabine in cervical cancer cells (HeLa). It was found that the incubation of cells with $5 \mu \mathrm{M}$ SFN resulted in a decrease in cell viability to $70 \%$ and with $15 \mu \mathrm{M}$ of gemcitabine to $75 \%$ as compared to the control cells. The compounds given in combination at such 
concentrations caused a decrease in HeLa cell viability up to $40 \%$. The same effect was also observed for the compounds at other concentrations, e.g., $2.5 \mu \mathrm{M}$ SFN in combination with 15 and $25 \mu \mathrm{M}$ gemcitabine. Studies on the mechanism of synergistic interaction of the compounds have shown an increase in apoptosis by significantly lowering the expression of antiapoptotic Bcl-2 protein and inflammatory proteins COX-2 (cyclooxygenase 2) and IL$1 \beta$ (Interleukin-1 $\beta$ ) after the SFN treatment (45).

A similar mechanism of synergistic action of SFN and gemcitabine have been demonstrated in studies on the combination of the compounds in breast cancer cells MCF-7. These results indicate that SFN induces apoptosis and inhibits proinflammatory pathways in MCF-7 cells by lowering the expression of Bcl-2 and COX-2, respectively (Table 1) (46). Moreover, studies using normal cell lung fibroblasts V-79 from the Chinese hamster showed the antagonistic effects of SFN and 5-FU, which evidenced that the combination does not exacerbate one another's toxicity in normal cells (5).

\section{ITC and 4-hydroxytamoxifen}

Tamoxifen inhibits competitive binding of estrogens to hormonal receptors in the cytoplasm, thereby limiting cellular divisions in estrogendependent tissues. It is used in adjuvant treatment of primary breast cancer and advanced metastatic breast cancer (47).

The research conducted by Pawlik et al. on the combined use of ITCs and tamoxifen was aimed at sensitizing breast cancer cells to the drug, as well as reducing tamoxifen systemic toxicity. The studies were conducted on the breast cancer cells (ER-positive) T47D, MCF-7 and BT-474, as well as tamoxifen-resistant T47D and MCF-7 cells. It has been shown that the combination of SFN or erucin and tamoxifen reduced the viability of breast cancer cells more effectively than any of the compounds used alone. This effect was due to a decrease in anti- and proapoptotic $\mathrm{Bcl}-2 / \mathrm{Bax}$ protein ratio, a reduction in the survivin levels, increased PARP cleavage and the elevated concentration of ADRP-mitochondrial stress marker. Moreover, the combination of $500 \mathrm{nM}$ tamoxifen and SFN or erucin statistically significantly reduced the viability of T47D and MCF-7 cells resistant to tamoxifen. The strategy of combining has allowed for the reduction of the dose while maintaining a complete therapeutic response (Table 1) (48).

\section{ITC and lapatinib}

Lapatinib is an antineoplastic agent from the group of protein kinase inhibitors. Lapatinib an inhibits the activity of tyrosine kinase which is a part of the epidermal growth factor receptor (EGFR) and epidermal growth factor receptor 2 (HER2). The inhibition of the activity of these kinases by lapatinib stops cell division and leads to apoptotic death of the cancer cell. The formulation is indicated for the treatment of patients with breast cancer overexpressing HER2 receptor (ErbB2) (49).

Recent research into the combined use of lapatinib with SFN and erucin conducted in breast cancer cells sensitive (SKBR-3) and resistant to lapatinib (SKBR-3 LapR) have shown the relevance of the combination of these compounds. The combination of lapatinib and SFN or erucin significantly reduced cell viability and inhibited the migration of populations consisting of a various number of lapatinib-sensitive and resistant cells. In the population composed of lapatinib-resistant cells, the combination of lapatinib and erucin was most effective in reducing the viability and invasiveness of cells and Protein Kinase B (AKT), ribosomal S6 kinase (S6) and Vascular endothelial growth factor (VEGF) phosphorylation was stronger than after separate administration of each compound. The combination of lapatinib and ITC, especially erucin, was regarded as promising treatment limiting the spread of breast cancer, even in cases of drug resistance phenotype (Table 1) $(50,51)$.

\section{ITC in paclitaxel and docetaxel therapy}

Paclitaxel and docetaxel induce apoptosis by inhibiting cell division on the meta- and anaphase boundary. Their mechanism of action is to stabilize the microtubules by preventing their depolymerization, which blocks reorganization of the microtubule network and consequently inhibits cell division. Paclitaxel is the first-line drug in the treatment of ovarian cancer; it is also used in a combined therapy of breast cancer and non-small cell lung cancer. Moreover, it is applied to treat oesophageal, bladder, head and neck and cervical malignancies (52). Docetaxel has been used mainly in the combined treatment of advanced breast cancer, metastatic breast cancer and hormone-dependent metastatic prostate cancer (53).

The studies of PEITC with paclitaxel in hormone-dependent MCF-7 and triple-negative breast cancer (TNBC) MDA-MB-231 cells have demonstrated strong antitumor activity of the combination which led to the significant reduction in doses of the compounds used in combination compared to their alone administration. PEITC $\mathrm{IC}_{50}$ for MCF-7 lines was 5.6 $\mu \mathrm{M}$, while $111.3 \mathrm{nM}$ for paclitaxel. After the administration of the compounds in combina- 
tion, $\mathrm{IC}_{50}$ for paclitaxel dropped to $3 \mathrm{nM}$ and for PEITC to $2.5 \mu \mathrm{M}$. For the MDA-MB-231 line, PEITC $\mathrm{IC}_{50}$ was $15.7 \mu \mathrm{M}$ and $409.6 \mathrm{nM}$ for paclitaxel. For the combination, $\mathrm{IC}_{50}$ for PEITC decreased to $1.9 \mu \mathrm{M}$ and to $8.1 \mathrm{nM}$ for paclitaxel. The synergistic effect of the compounds has been explained by an increase in the number of the cells in the G2/M cell cycle phase. Studies indicated that PEITC significantly reduces the dosage of cytostatic, which is associated with lower systemic toxicity of paclitaxel (27).

Similar results have been obtained in the studies of the interaction between SFN and paclitaxel performed in ovarian carcinoma epithelial cells MDAH 2774 and SkOV-3. The synergistic effect was due to cell migration inhibition and apoptosis induction by increasing the $\mathrm{Bax} / \mathrm{Bcl}-2$ protein level ratio. The combination of the compounds also induced the cell cycle arrest in the G1 phase (Table 1) (54).

The studies with PEITC and docetaxel were also performed in in vitro and in vivo prostate tumor models using the PC-3, DU-145 lines and PC-3 xenograft mice. The combination of docetaxel and PEITC at $1 \mu \mathrm{M}$ non-toxic concentration and $2 \mu \mathrm{M}$ cytotoxic concentration synergistically reduced the viability of prostate tumor cells. The effect observed in the PC-3 cells was stronger than in DU-145 cells. The induction of apoptosis after combination treatment was associated with the lowered Bcl-2 levels and increased concentrations of Bax and Bak proteins in the cells. Moreover, in vivo studies have shown that the combination was well tolerated (55).

The recent research conducted by Burnett et al. regarding TNBC therapy revealed that the combination of SFN and paclitaxel or docetaxel reduced the expansion of breast cancer stem cells (CSC). Expansion of these cells may lead to ineffective therapy, metastasis promotion, and induction of relapse. Paclitaxel and docetaxel therapy has been shown to elevate the CSC levels by increasing IL-6 levels. The research conducted by Brunett et al. has demonstrated that SFN reversed this process and prevented CSC expansion by inhibiting NF- $\mathrm{KB}$ which regulates the production of proinflammatory cytokines. SFN has also been found to reduce the expression of p52 protein. In vivo studies showed, in turn, a larger reduction in the primary tumor volume, as well as a decrease in the incidence of secondary tumors following the combined therapy compared to the treatment with the cytostatic alone. The results suggest that TNBC treatment would be greatly improved by combining SFN with standard chemotherapy to prevent breast CSC expansion (Table 1) (56).

\section{ITC and temozolomide}

Temozolomide (TMZ) is used to treat glioblastoma multiforme (GBM) in monotherapy or in combination with radiotherapy. It belongs to alkylating compounds. The active metabolite is 3-methyl-(triazen-1-yl) imidazole-4-carboxamide (MTIC), which spontaneously hydrolyzes to 5-aminoimidazole-4carboxamide (AIC), an intermediate compound involved in biosynthesis of purines and nucleic acids, and to methylhydrazine, is considered to be a proper alkylating compound. Due to the acquired resistance to TMZ, the percentage of cured patients with GBM is still unsatisfactory. Studies have shown that TMZ resistance in GBM therapy is associated with the O6-methylglycin methyltransferase (MGMT), which elevated activity may lead to DNA replication and GBM cell growth (57).

The research conducted by Lan et al. examined the TMZ combination with SFN to sensitize TMZresistant glioblastoma cells U87-R and U373-R. It has been shown that the sequential combination of $250 \mu \mathrm{M}$ TMZ and SFN at concentrations of 5-30 $\mu \mathrm{M}$ synergistically inhibited the GBM cell growth, which reduced the dosage of the drug used from about 2 to as much as 26 times. In addition, SFN effectively inhibited the activity of the NF- $\mathrm{KB}$ signaling pathway and reduced the expression of MGMT, resulting in the reduction of TMZ resistance in the T98G, U87-R, and U373-R cell lines. Significantly, studies in mice with subcutaneously implanted U373-R tumor cells showed that this combination significantly reduced tumor cell growth. The combination of compounds also induced in vitro and in vivo apoptosis by increasing the caspase- 3 levels and decreasing the expression of Ki-67, MMP2/6 - proteins involved in cell proliferation and migration. Studies suggest that SFN may become a clinically effective cell sensitizer to TMZ (Table 1) (58).

\section{CONCLUSION}

ITCs, which are found in the vegetables from the Brassicaceae family, are considered to have high antitumor potential; therefore the number of studies using these compounds is constantly increasing. In many of the examples described above, the use of ITC combinations with classic chemotherapeutic agents, not only potentiated cytotoxic or cytostatic effects, but also allowed for reduction of the dosage of the drugs used and, in turn, reduced the frequen- 
cy and severity of adverse effects. The in vitro and in vivo studies have shown that the use of ITC with standard anticancer therapy has increased the antitumor effect, reduced tumor mass, and was of minimal toxicity to normal cells. The mechanism responsible for enhancing the cytotoxic effect may be different, depending on the combinations of compounds used, dosages, experiment's design, incubation time and cell line tested. Taking into account the examples quoted in this article, including ITC into the therapy may present a new opportunity for cancer treatment. The combination of ITCs with immunotherapeutics which include inhibitors of kinases (eg. lapatinib) seem to be particularly promising. Similarly, SFN was shown to sensitize breast cancer cells resistant to tamoxifen or to increase the effectiveness of TNBC therapy, which remains a challenge for clinicians.

Simultaneously the literature data indicate that the impact of ITC on the activity of I, II and III xenobiotics metabolism phase enzymes may cause clinical implications. As described in many publications, ITC can induce faster elimination of drugs from the cell by inducing enzymes, such as NQO1, GST, or proteins belonging to MRP family, thereby weakening the therapeutic effect. This phenomenon can also contribute to the development of multidrug resistance. These examples point to the importance of the choice of therapy depending on the type of cancer and indicate the role that ITCs can play as enzyme inducers, leading to the increased therapeutic efficacy. In other cases, they suggest the unfavourable role of ITC-rich diet as leading to therapeutic resistance.

Despite the high hopes of combined therapy with the use of ITC and growing interest among researchers in this topic, there is still no data on the impact of ITC on the pharmacokinetics of chemotherapeutics. It is necessary to carry out more in vivo studies on the ITC combination with drugs assessing the effect of the combination on tumor and normal tissue, in order to confirm the efficacy of ITC in antitumor politherapy. Particular attention should also be paid to the effect of the combination on the activity xenobiotics metabolism phase enzymes.

\section{Conflict of interest}

None declared.

\section{REFERENCES}

1. Bernard W.S., Christopher P.W.: World Cancer Report 2014; International Agency for Research on Cancer World Health Organization. 2014.
2. Doudican N.A., Bowling B., Orlow S.J.: Leuk. Res. 34, 229 (2010).

3. Lee Y., Kim Y.J., Choi Y.J., Lee J.W., Lee S. et al.: Food Chem. Toxicol. 50, 2397 (2012).

4. Li D., Wang W., Shan Y., Barrera L.N., Howie A.F. et al.: Food Chem. 133, 300 (2012).

5. Milczarek M., Misiewicz-Krzemińska I., Lubelska K., Wiktorska K.: Acta Pol. Pharm. 68, 331 (2011).

6. Mukherjee S., Dey S., Bhattacharya R.K., Roy M.: Mol. Cell. Biochem.: 330, 9 (2009).

7. Śliwka L., Wiktorska K., Suchocki P., Milczarek M., Mielczarek S. et al.: PLoS ONE 11(5), e0155772 (2016).

8. Wang X.F., Wu D.M., Li B.X., Lu Y.J., Yang B.F.: Phytother. Res. 23, 303 (2009).

9. Wolf M.A., Claudio P.P.: Nutr. Cancer. 66, 285 (2014).

10. Leslie E.M., Deeley R.G., Cole S.P.C.: Toxicol. Appl. Pharmacol. 204, 216 (2005).

11. De Larco J.E., Park C.A., Dronava H., Furcht L.T.: Cancer Biol. Ther. 9, 362 (2010).

12. Zeekpudsa P., Kukongviriyapan V., Senggunprai L., Sripa B., Prawan A.: J. Exp. Clin. Cancer Res. 33, 11 (2014).

13. Maher J.M., Dieter M.Z., Aleksunes L.M., Slitt A.L., Guo G. et al.: Hepatology 46, 1597 (2007).

14. Harris K.E., Jeffery E.H.: J. Nutr. Biochem. 19, 246 (2008).

15. Lubelska K., Milczarek M., Modzelewska K., Krzysztoń-Russjan J., Fronczyk K. et al.: Pharmacol. Rep. 64, 1243 (2012).

16. Lubelska K., Misiewicz-Krzemińska I., Milczarek M., Krzysztoń-Russjan J., Anuszewska E. et al.: Mol. Cell. Biochem. 367, 19 (2012).

17. Zhou C., Poulton E.J., Grün F., Bammler T.K., Blumberg B. et al.: Mol. Pharmacol. 71, 220 (2007).

18. Reinicke K.E., Bey E.A., Bentle M.S., Pink J.J., Ingalls S.T. et al.: Clin. Cancer Res. 11, 3055 (2005).

19. Qazi A., Pal J., Maitah M., Fulciniti M., Pelluru D. et al.: Transl. Oncol. 3, 389 (2010).

20. Tseng E., Kamath A., Morris M.E.: Pharm. Res. 19, 1509 (2002).

21. Finley J.W., Sigrid-Keck A., Robbins R.J., Hintze K.J.: J. Nutr. 135,1236 (2005).

22. Blum N.M., Mueller K., Lippmann D., Metges C.C., Linn T. et al.: Biol. Trace Elem. Res. 151, 384 (2013).

23. Campbell L., Howie F., Arthur J.R., Nicol F., Beckett G.: Nutr. 23, 138 (2007).

24. Wang Y., Dacosta C., Wang W., Zhou Z., Liu M.: Nutr. Res. 35, 610 (2015). 
25. Zhang J., Svehlíková V., Bao Y., Howie A.F., Beckett G.J. et al.: Carcinogenesis 24, 497 (2003)

26. Chen H., Landen C.N., Li Y., Alvarez R.D., Tollefsbol T.O.: J. Oncol. 2013, 872957 (2013).

27. Liu K., Cang S., Ma Y., Chiao J.W.: Cancer Cell Int. 13, 10 (2013).

28. Kaminski B.M., Weigert A., Brüne B., Schumacher M., Wenzel U. et al.: Cancer Chemother. Pharmacol. 67, 1167 (2011).

29. Murgo A.J.: The Oncologist. 6, 22 (2001).

30. Doudican N.A., Wen S.Y., Mazumder A., Orlow S.J.: Oncol. Rep. 28, 1851 (2012).

31. Roccaro A.M., Vacca A., Ribatti D.: Recent Patents Anticancer Drug Discov. 1, 397 (2006).

32. Jakubikova J., Cervi D., Ooi M., Kim K., Nahar S. et al. Haematologica 96, 1170 (2011).

33. Wang X., Govind S., Sajankila S.P., Mi L., Roy R. et al.: Mol. Nutr. Food Res. 55, 1572 (2011).

34. Wang X., Govind S., Sajankila S.P., Mi L., Roy R. et al.: Mol. Nutr. Food Res. 55, 1572 (2011).

35. Di Pasqua A.J., Hong C., Wu M.Y., McCracken E., Wang X. et al.: Chem. Res. Toxicol. 23, 1307 (2010).

36. Gaona-Gaona L., Molina-Jijón E., Tapia E., Zazueta C., Hernández-Pando R. et al.: Toxicology. 286, 20 (2011).

37. Lüpertz R., Wätjen W., Kahl R., Chovolou Y.: Toxicology. 271, 115 (2010).

38. Fimognari C., Nüsse M., Lenzi M., Sciuscio D., Cantelli-Forti G. et al.: Mutat. Res. 601, 92 (2006).

39. Mukherjee S., Bhattacharya R.K., Roy M.: J. Environ. Pathol. Toxicol. Oncol. 28, 269 (2009).

40. Mazur M., Krug P., Wiktorska K., Chilmończyk Z., Śliwka L.: Polish Patent PL414021 (A1) March, 27 (2017)

41. Li B., Kim D.S., Yadav R.K., Kim H.R., Chae H.J.: Int. J. Mol. Med. 36, 53 (2015).

42. Singh P., Sharma R., McElhanon K., Allen C.D., Megyesi J.K. et al.: Free Radic. Biol. Med. 86, 90 (2015).
43. Longley D.B., Harkin D.P., Johnston P.G.: Nat. Rev. Cancer. 3, 330 (2003).

44. Toschi L., Finocchiaro G., Bartolini S., Gioia V., Cappuzzo F.: Future Oncol. 1, 7 (2005).

45. Sharma C., Sadrieh L., Priyani A., Ahmed M., Hassan A.H., et al.: Cancer Epidemiol. 35, 272 (2011).

46. Hussain A., Mohsin J., Prabhu S.A., Begum S., Nusri Q.E.A, et al.: Asian Pac. J. Cancer Prev. 14, 5855 (2013).

47. Jordan V.C.: Curr. Probl. Cancer. 16, 129 (1992).

48. Pawlik A., Słomińska-Wojewódzka M., Herman-Antosiewicz A.: Eur. J. Nutr. 55, 1165 (2016).

49. Blackwell K.L., Burstein H.J., Storniolo A.M., Rugo H., Sledge G. et al.: J. Clin. Oncol. 28, 1124 (2010).

50. Kaczyńska A., Herman-Antosiewicz A.: Breast Cancer. 24, 271 (2017).

51. Kaczyńska A., Świerczyńska J., HermanAntosiewicz A.: Nutr. Cancer 67, 976 (2015).

52. Mekhail T.M., Markman M.: Expert Opin. Pharmacother. 3, 755 (2002).

53. Crown J., O’Leary M., Ooi W.S.: Oncologist 9, 24 (2004).

54. Bryant C.S., Kumar S., Chamala S., Shah J., Pal J. et al.: Mol. Cancer 9, 47 (2010).

55. Xiao D., Singh S.V.: Pharm. Res. 27, 722 (2010).

56. Burnett J.P., Lim G., Li Y., Shah R.B., Lim R. et al.: Cancer Lett. 394, 52 (2017).

57. Friedman H.S., Kerby T., Calvert H.: Clin. Cancer Res. 6, 2585 (2000).

58. Lan F., Yang Y., Han J., Wu Q,. Yu H. et al.: Int. J. Oncol. 48, 559 (2016).

59. Minarini A., Milelli A., Fimognari C., Simoni E., Turrini E. et al.: Expert Opin. Drug Metab. Toxicol. 10, 25 (2014).

Received: 14.09. 2017 